\title{
EXTENSIONS OF HAAR MEASURE FOR COMPACT CONNECTED ABELIAN GROUPS
}

\author{
BY GERALD L. ITZKOWITZ ${ }^{1}$ \\ Communicated by E. Hewitt, August 21, 1964
}

We outline in this paper generalizations of some theorems of Hulanicki on the existence of dense subsets of small cardinality in product measure spaces and in compact groups. We then apply a special case of these results to show the existence of the Kakutani-Oxtoby measure for the case of compact connected Abelian topological groups. A more detailed paper will appear later on.

Definition. Let $a, \beta$ be collections of nonvoid sets of a space $X$. Then $Q$ is a weak base for $B$ if and only if given $B \in B$ there is an $A \in Q$ such that $A \subset B$.

If $A$ is a set then $|A|$ denotes the cardinal of $A ; \mathfrak{n}$ will always denote an infinite cardinal.

The following theorem generalizes Hulanicki [7, Theorem 1].

Theorem 1. Let $X=P_{t \in T} X_{t}$, where $\left\{\left(X_{t}, \Theta_{t}\right): t \in T\right\}$ is a family of measurable spaces, each having a weak base of cardinal at most $n \boldsymbol{N}_{0}$, and $|T| \leqq 2^{\mathrm{n}}$. Then the product measurable space $(X, B)$ has a weak base a for the $\sigma$-field $B$ for which $|a| \leqq \mathfrak{n N}$.

The proof uses the following lemma.

Lemma 1. Let $T$ be any set such that $|T|=2^{\mathfrak{n}}$; then there exists a family $\mathfrak{A}$ of sequences $\left\{B_{i}\right\}_{i=1}^{\infty}$ of pairwise disjoint subsets of $T$ such that

(i) $|\mathfrak{A}| \leqq \mathfrak{n} \mathbf{N}^{0}$,

(ii) for any distinct sequence $\left\{t_{i}\right\}_{i=1}^{\infty}$ in $T$, there exists a sequence $\left\{B_{i}\right\}_{i=1}^{\infty} \in \mathcal{Q}$ such that $t_{i} \in B_{i}$ for each $i$.

This lemma can be proved by noticing that there is a 1-1 correspondence of $T$ with $\{-1,1\}^{\mathfrak{n}}$, and this latter set has at most $\mathfrak{n}$ No closed $G_{\delta}$ sets.

Let $X$ be a topological space. Let $w(X)$ denote the least cardinal of a basis of open sets for $X$. It is not difficult to show that if $H$ is a compact Abelian group and if $w(H) \leqq \mathfrak{n}$, then $H$ has at most $\mathfrak{n} \boldsymbol{N}_{0}$ closed $G_{\delta}$ sets. Thus, trivially, there is a weak base for the Baire sets of $H$ having cardinal at most $\mathfrak{n}$.

${ }^{1}$ This research was supported in part by an NSF fellowship. It will be used in partial fulfillment of the requirements for the $\mathrm{Ph} . \mathrm{D}$. at the University of Rochester. I wish to thank Professor K. A. Ross for the suggestion of the problem in the title of this paper and for his invaluable guidance and criticism. 
Corollary 1. Let $G=\boldsymbol{P}_{t \in T} H_{t}$, where each $H_{t}=H, H$ is a compact Abelian group, $w(H) \leqq \mathfrak{n}$, and $|T| \leqq 2^{\mathfrak{n}}$. Then $w(G) \leqq 2^{\mathfrak{n}}$ and there is a weak base for the Baire sets of $G$ having cardinal at most $\mathfrak{n}$ No.

Kakutani [8] has shown that if $w(H)=\mathfrak{n}$, then $|H|=2^{\mathfrak{n}}$ (see also $[3,24.47])$. Thus the following holds.

Corollary 2. Let $G=P_{t \in T} H_{t}$, where each $H_{t}=H, H$ is a compact Abelian group, $w(H) \leqq \mathfrak{n}$, and $|T| \leqq 2^{2^{\mathfrak{n}}}$. Then there is a weak base for the Baire sets of $G$ having cardinal at most $2^{\text {n }}$.

Corollary 3. Let $G$ be as in Corollary 2. Then $G$ has a dense pseudocompact subgroup $J$ which necessarily has Haar outer measure one and $|J| \leqq 2^{\mathfrak{n}}$.

This follows from a theorem of Comfort and Ross [1] which states that a totally bounded group $G$ is pseudocompact if and only if each nonempty Baire subset of $\bar{G}$ meets $G$, where $\bar{G}$ is the Weil completion of $G$. We note in the proof that if $H$ is a compact group and if $A \subset H$, then $A$ has Haar outer measure one if and only if $A \cap B \neq \varnothing$ for each Baire set $B$ of positive measure.

TheOREM 2. Let $G$ be a compact Abelian topological group satisfying $w(G)=2^{\mathfrak{n}}$ for some infinite cardinal number $\mathfrak{n}$. Then

(i) G has a weak base for its Baire sets of cardinal at most $\mathfrak{n} N$,

(ii) $G$ contains a dense pseudocompact subgroup $J$ such that $|J|$ $\leqq \mathfrak{n}$; necessarily $J$ has outer measure one.

This theorem is proved by using Corollary 1 and the following theorem of Vilenkin [11]: Let $G$ be a compact Abelian group. For some cardinal number $\mathfrak{m}$, there is a continuous mapping of $\{-1,1\}^{\mathfrak{m}}$ onto $G ; \mathfrak{m}$ can be taken to be $\max \left[\boldsymbol{\aleph}_{0}, r\right]$, where $r$ is the rank of the character group of $G$.

We may observe that Theorem 2 is a generalization of a theorem of Hartman and Hulanicki [2]: If $G$ is a compact group satisfying $|G| \leqq 2^{2^{n}}$ and if the generalized continuum hypothesis holds, then there is a dense subgroup $H \subset G$ satisfying $|H| \leqq \mathfrak{n}$. We note here that we did not use the generalized continuum hypothesis. Finally, part (i) of Theorem 2 appears to contain Theorem 2 of Hulanicki [7].

We next prove a special case of Corollary 2. We note that Corollary 2 is an existence theorem. We will now construct a set that is actually a weak base for the closed $G_{\delta}$ sets of $G$ in Corollary 2.

Let $G$ be as in Corollary 2. Let $\Re$ be the collection of closed $G_{\delta}$ sets of $H$. As above we note that $|\mathfrak{x}| \leqq n N_{0}$. Let $\mathfrak{A}$ be the collection of sequences of pairwise disjoint sets in $T$ satisfying (i) and (ii) of Lemma 1. 
Definition. An ( $\mathfrak{A}, \mathfrak{r})$-cylinder set in $G$ is a set of the form $M=\bigcap_{i=1}^{\infty}\left\{\bigcap_{t(i) \in B(i)} \pi_{t(i)}^{-1}\left(N_{t(i)}\right)\right\}$, where $\{B(i)\}_{i=1}^{\infty} \in \mathfrak{A}$, and for each $i$, all $N_{t(i)}=N_{i}$ for some $N_{i} \in \Re$.

Let $\mathfrak{C}_{\delta}$ be the collection of all $(\mathfrak{A}, \mathfrak{\Re})$-cylinder sets in $G$. It is immediate from Lemma 1 that $\left|\mathfrak{C}_{\boldsymbol{\delta}}\right| \leqq 2^{\mathfrak{n}}$.

Theorem 3. Let $G$ be as in Corollary 2. Then $\mathfrak{C}_{\delta}$ is a weak base for the closed $G_{\delta}$ sets in $G$.

The proof of this theorem uses the following lemma and the reflexivity of the property of being a weak base.

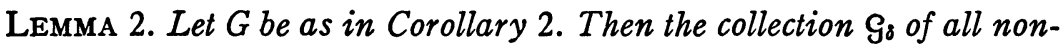
void closed $G_{\delta}$ sets in $G$ of the form $\bigcap_{i=1}^{\infty} \pi_{t(i)}^{-1}\left(N_{t(i)}\right)$, where $\{t(i)\}_{i=1}^{\infty} \subset T$, and $N_{t(i)} \in \Re$ for each $i$, is a weak base for the closed $G_{\delta}$ sets in $G$.

Kakutani and Oxtoby [10] proved that Haar measure in a compact metric group may be extended to a much larger $\sigma$-field of subsets of the group and still remain invariant under group translation and inversion. To be more precise we introduce the following definition.

Definition. The character of a measure space $(X, \delta, \mu)$ is the smallest cardinal number $\mathfrak{m}$ for which there is a subfamily $R \subset S$ such that $|R|=\mathfrak{m}$ and such that for each $S \in \mathcal{S}$ and each $\epsilon>0$, there exists a set $R \in R$ satisfying $\mu(S \triangle R)<\epsilon$.

It is well known that the character of the Haar measure space of a compact infinite metric group is $\boldsymbol{\aleph}_{0}$. Kakutani and Oxtoby showed that there is an extension of Haar measure with character $2^{c}$.

Kakutani and Kodaira [9] showed that there is an extension of Haar measure on the circle of character c. Hulanicki [7], using Theorem 1 of his paper, showed that the method of Kakutani and Kodaira may be used to get an extension of character $2^{c}$.

TheOREM 4. Let $H$ be a compact connected Abelian topological group satisfying $w(H)=\mathfrak{n}$. Then there exists a translation- and inversioninvariant extension of Haar measure on $H$ of character $2^{2^{n}}$.

We remark that for a compact infinite Abelian group $G$ it is easy to show that the character of the Haar measure space of $G$ is equal to $w(G)$. Thus the character of the Haar measure space of $H$ in the above theorem is $\mathfrak{n}$. Our method of proof of Theorem 4 is similar to that of Kakutani and Kodaira. We briefly outline the proof in the following theorem and lemmas.

Theorem 5. Let $G$ be as in Corollary 2. Let $\beta \in T$ be fixed. Let

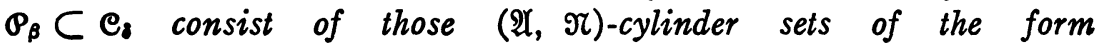


$\bigcap_{n=1}^{\infty}\left\{\bigcap_{t(n) \in B(n)} \pi_{t(n)}^{-1}\left(N_{n}\right)\right\}$ that satisfy $\beta \in B(i)$ for some $i$ and $N_{i}$ has positive Haar measure in $H$ for this $i$. Then $\odot_{\beta}$ is a weak base for the closed $G_{\delta}$ sets in $G$ having positive Haar measure.

REMARK. It is clear from the construction of $\mathcal{P}_{\beta}$ that $\left|\mathcal{\odot}_{\beta}\right| \leqq 2^{\mathfrak{n}}$ and if $A \in \mathcal{P}_{\beta}$ then $\pi_{\beta}(A)$ has positive Haar measure in $H_{\beta}$ and is a closed $G_{\delta}$ there $\left(\pi_{\beta}\right.$ is the projection onto $\left.H_{\beta}\right)$.

Lemma 3. Let $G$ be a compact Abelian topological group. Let $M \subset G$ be a set of positive Haar measure. Then $M$ contains a maximal independent set of elements of infinite order in $G$.

This lemma is a consequence of a well-known theorem which states (using additive notation) that if $M$ has positive Haar measure in $G$ then $M-M$ contains the identity in its interior. It follows then that the group $[M]$ generated by $M$ has finite index in $G$ if $G$ is compact and hence every element of infinite order in $G$ is dependent on $M$.

Lemma 4. Let $G$ be a compact connected Abelian topological group satisfying $w(G)=\mathfrak{n}$. Then every closed $G_{\delta}$ set $M \subset G$ having positive Haar measure contains a maximal linearly independent set $L$ of elements of infinite order in $G$ and $|L|=2^{\mathfrak{n}}$.

This lemma follows from Lemma 3 , the fact that all maximal linearly independent sets of elements of infinite order have the same cardinality, and a structure theorem of Hulanicki [5], [6] for compact connected Abelian groups. Lemma 4 allows us to carry out a transfinite induction which leads to:

Lemma 5. Let $G$ be a compact connected Abelian group satisfying $w(G)=\mathfrak{n} \geqq \boldsymbol{\aleph}_{0}$. Let $\left\{M_{\alpha}: \alpha<\omega_{\mathfrak{m}}, \mathfrak{m}=2^{\mathfrak{n}}\right\}$ be a well-ordered sequence of closed $G_{\delta}$ sets of positive Haar measure in $G$. Then there exists a wellordered set $\left\{x_{\alpha}: \alpha<\omega_{\mathfrak{m}}\right\}$ of independent elements of infinite order such that $x_{\alpha} \in M_{\alpha}$ for each $\alpha<\omega_{\mathfrak{m}}$. (The $M_{\alpha}$ 's are not necessarily distinct.)

REMARK. Lemma 5 is true in a more general situation. The same induction will work because of Lemma 3 if the $M_{\alpha}$ are measurable with positive measure, $\mathfrak{m}$ is at most equal to the cardinal of a maximal independent set of elements of infinite order, and $G$ is compact Abelian (with no other restrictions).

Lemma 6. Let $H$ be a compact connected Abelian group satisfying $w(H)=\mathfrak{n} \geqq \boldsymbol{\aleph}_{0}$. Let $G=\boldsymbol{P}_{t \in T} H_{t}$ where each $H_{t}=H$ and $|T|=2^{2^{\mathfrak{n}}}$. Fix the coordinate $\beta \in T$. Then there is a set $V \subset G$ of independent elements of infinite order satisfying

(i) V has Haar outer measure one,

(ii) $\left.\pi_{\beta}\right|_{V}$ is one-to-one. 
This is proved by using Theorem 5 (i.e., projecting onto $H_{\beta}$ the elements of $\mathcal{P}_{\beta}$ ) and then using Lemma 5.

Letting $V_{G}$ be the free group generated by $V$, and letting $W$ be the free group generated by $\pi_{\beta}(V)$, it is easy to see that $\pi_{\beta}$ induces an algebraic isomorphism $\phi$ of $W$ onto $V_{G}$. Furthermore, $\phi$ may be extended to an algebraic isomorphism of $H$ into $G$ satisfying $\pi_{\beta} \phi(x)=x$ for all $x \in H_{\beta}$, because $H$ and $G$ are divisible. It follows that $\phi\left(H_{\beta}\right)$ is a group of outer measure one in $G$. Thus the remainder of the proof of Theorem 4 is a repetition of the final part of the proof of Kakutani and Kodaira [9] for the circle.

REMARK. One could use the method of proof outlined above without Theorem 5 to show the existence of an extension of Haar measure of character $2^{\mathfrak{n}}$.

Note. Since this work was completed, Hewitt and Ross [4], have generalized and simplified Theorem 4; their theorem implies Theorem 4 for all compact Abelian groups, and uses our Theorem 2, Lemma 3, and Lemma 5 with the remark following it.

\section{BIBLIOGRAPHY}

1. W. W. Comfort and K. A. Ross, Pseudocompactness and uniform continuity in topological groups (to appear).

2. S. Hartman and A. Hulanicki, Sur les ensembles denses de puissance minimum dans les groupes topologiques, Colloq. Math. 6 (1958), 187-191.

3. E. Hewitt and K. A. Ross, Abstract harmonic analysis, Vol. I, Springer-Verlag, Heidelberg, 1963.

4. - Extensions of Haar measure and of harmonic analysis for locally compact Abelian groups, Math. Ann. (to appear).

5. A. Hulanicki, Algebraic characterization of abelian divisible groups which admit compact topologies, Fund. Math. 44 (1957), 192-197.

6. - Algebraic structure of compact abelian groups, Bull. Acad. Polon. Sci. Sér. Sci. Math. Astronom. Phys. 6 (1958), 71-73.

7. - On subsets of full outer measure in products of measure spaces, Bull. Acad. Polon. Sci. Sér. Sci. Math. Astronom. Phys. 7 (1959), 331-335.

8. S. Kakutani, On cardinal numbers related with a compact abelian group, Proc. Imp. Acad. Tokyo 19 (1943), 366-372.

9. S. Kakutani and $\mathrm{K}$. Kodaira, $A$ non-separable translation invariant extension of the Lebesgue measure space, Ann. of Math. (2) 52 (1950), 574-579.

10. S. Kakutani and J. C. Oxtoby, Construction of a non-separable invariant extension of the Lebesgue measure space, Ann. of Math. (2) 52 (1950), 580-590.

11. N. Ja. Vilenkin, On the dyadicity of the group space of bicompact commutative groups, Uspehi Mat. Nauk (N.S.) 6 (84) (1958), 79-80.

UNIVERSITY OF ROCHESTER 